\title{
Manufacturing of Sardine Terrine by Addition of Carrageenan*1
}

\author{
Teruo Nakayama, ${ }^{* 2}$ Yoshihito Kashiwagi, ${ }^{* 2}$ Satoshi Kanoh, ${ }^{* 2}$ \\ and Eiji Niwa*2
}

(Accepted September 8, 1987)

\begin{abstract}
Sardine terrine was produced from minced sardine and carrageenan. The addition of $\kappa$ carrageenan increased jelly strength markedly and breaking deformation slightly. But the excessive addition of $\kappa$-carrageenan decreased clearly breaking deformation of product from dressed sardine. The addition of -carrageenan increased jelly strength moderately, but did not change breaking deformation. The addition of $\lambda$-carrageenan increased jelly strength very gently, but decreased breaking deformation. The result of the sensory evaluation corresponded to the instrumental values of jelly strength. Panelists favored the products with $\kappa$-carrageenan and $t$-carrageenan in both cases of products from round and dressed fish. Among them, the product from dressed fish with $6 \% \kappa$-carrageenan was most favorable. This result would be due to the dropping of larger force after first peak. All sardine terrines were juicy, having the moisture of about $65 \%$. The gelation of minced sardine with $\kappa$ - or i-carrageenan proceeded at the temperature below about $80^{\circ} \mathrm{C}$. The addition of $\lambda$-carrageenan to minced sardine did not induce the gelation owing to the decline of temperature. The properties of $k-, t-$, and $\lambda$-carrageenans reported previously, were revealed even if they were added to minced sardine. And the effects were more vivid when minces from dressed sardine were used.
\end{abstract}

Enormous quantity of sardine Sardinops melanosticta is landed along the Pacific coast of Japan. Since sardine contains various kinds of nutritive substances not only in muscle, but also in skin, bones, guts, etc., ${ }^{1,2)}$ some trial manufacturings of new products incorporated also these latter parts, have been made. Nakayama et al. produced sardine fibers ${ }^{3,4)}$ and restructed sardine meat ${ }^{53}$ from round or dressed sardine including skin and bones. In this case, sodium alginate was used to solidify minced sardine. On the other hand, carrageenans had been used to provide for food thickening by food producers. Cooked ham and poultry roll were processed with carrageenan which increases water binding capacity and improves texture. ${ }^{6)}$ In canned meat and fish products, carrageenan is incorporated for maintaining a gelled broth or coating. ${ }^{6)}$ Recently Rees, ${ }^{72}$ Morris et al. ${ }^{8>}$ reported schematic representation of the gelation in carrageenan, and Therkelsen ${ }^{\theta)}$ reported the solubility of carrageenans. In the present study, we tried to produce minced sardine jelly with carrageenan. And the physical properties of both the final products and the minced sardine during processing were investigated.

\section{Materials and Methods}

\section{Manufacturing Process of Sardine Terrine}

Round sardine for processing was frozen-stored at $-40^{\circ} \mathrm{C}$ and thawed just before use. Subsequent process was shown in Fig. 1. Sardine (round or dressed) was chopped through meat chopper, and ground four times by Polytron (PT 45/2 "OD" Kinematica Switzerland) dialling 6.8 for $5 \mathrm{~min}$ at each time. Carrageenans (food applications) were supplied by Taiyo Kagaku Co., Ltd., Yokkaichi, Japan. A given amount of $\kappa$, $\imath-$, or $\lambda$-carrageenan was mixed little by little with the ground sardine. The mixture was stufied into Krehalon casing (19.1 mm diameter), cooked in boiling water for $30 \mathrm{~min}$, cooled by tape water, and stored overnight at $5^{\circ} \mathrm{C}$ in the refrigerator for aging. This product was named sardine terrine after French cuisine.

\section{Measurement of Physical Properties of Sardine Terrine}

Textural properties of sardine terrine were evaluated in terms of jelly strength and breaking

*1 This work was supported by a Grant from the Ministry of Agriculture, Forestry and Fisheries.

*2 Faculty of Fisheries, Mie University, Tsu 514, Japan (中山照雄, 柏木良仁, 加納 哲, 丹羽栄二：三重大 学水産学部). 


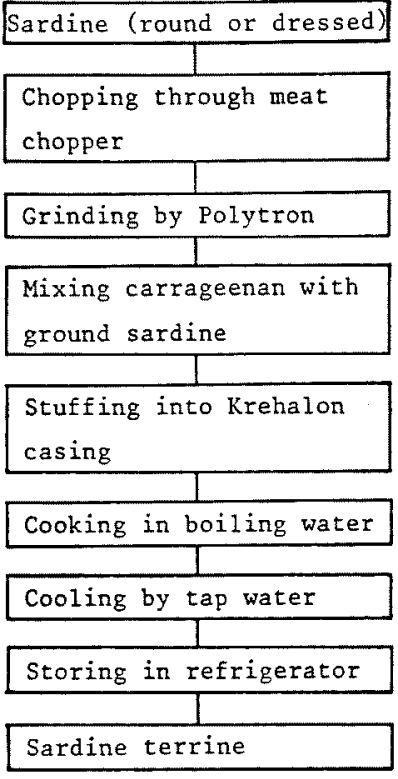

Fig. 1. Manufacturing process of sardine terrine.

deformation. A ball-point plunger ( $7 \mathrm{~mm}$ diameter) was fitted onto the Rheometer (Type NRM2010J-CW, Fudoh Kogyo Co., Ltd., Tokyo). The plunger was allowed to penetrate into the center of columnar sample of sardine terrine at penetration speed of $0.9 \mathrm{~mm} / \mathrm{s}$. Height and temperature of sample analyzed were $16.5 \mathrm{~mm}$ and $10.0 \pm$ $0.5^{\circ} \mathrm{C}$, respectively. Jelly strength and breaking deformation were expressed by the force $(\mathrm{g})$ and the deformation $(\mathrm{mm})$ measured at the first peak observed in force vs. deformation curve. The first peak is the point where there was an abrupt change in the slope of the force vs. deformation curve (See Fig. 5). ${ }^{5)}$

\section{Sensory Evaluation of Sardine Terrine}

Sensoty evaluation was done on the sense intensity of jelly strength. This property was evaluated by the sense felt when a piece of the sample was bitten off. Panel members independently reported their numerical judgments on a written ballot. A three-point category scale of intensity was employed, as follows: $1=$ weak, $2=$ medium, and $3=$ strong. Intermediate $(1 / 2$ point) designations were also used if necessary. Panelists additionally reported the impression about the odour on the ballot.

\section{Measurement of Physical Properties of Minced Sardine during Cooling Process}

Physical state of minced sardine with carrageenan changed from sol to gel while the temperature of minced sardine was lowered from $100^{\circ} \mathrm{C}$ to $5^{\circ} \mathrm{C}$ during cooling process shown in Fig. 1 . This change was monitored by means of the Rheolograph (Type 653, Toyo Seiki Mfg. Co., Ltd., Tokyo). Viscoelastic properties of minced sardine were evaluated in terms of stroage modulus $G^{\prime}$ and loss modulus $G^{\prime \prime}$. Strain amplitude was 0.10 , and frequency was $3 \mathrm{cycle} / \mathrm{s}$. The cooling rate of minced sardine was controlled approximately $5^{\circ} \mathrm{C} / \mathrm{min}$.
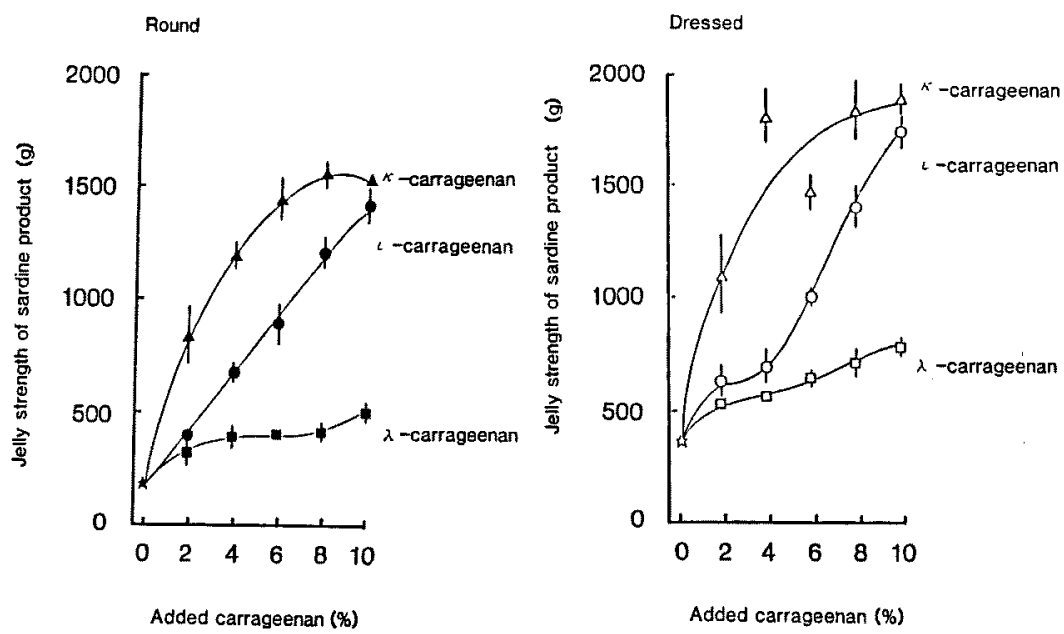

Fig. 2. Effect of type and concentration of carrageenan on jelly strength of sardine terrine. Proximate analysis of sardine mince: round; lipid $11.6 \%$, moisture $68.5 \%$, dressed; lipid $10.4 \%$, moisture $70.5 \%$. 

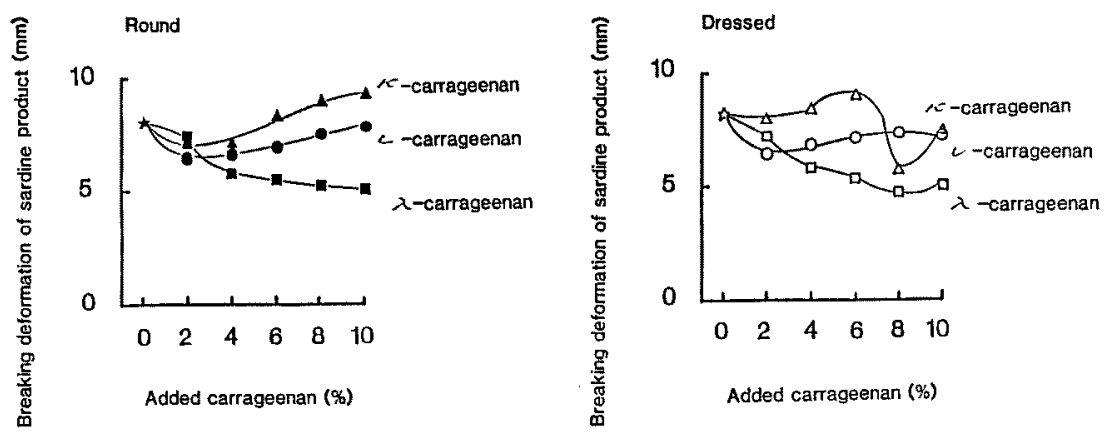

Fig. 3. Effect of type and concentration of carrageenan on breaking deformation of sardine terrine. Data were obtained from the same sardine terrine as shown in Fig. 2.

\section{Results and Discussion}

Effects of Type and Concentration of Carrageenan on Physical Properties of Sardine Terrine

Jelly strength of sardine terrine is shown in Fig. 2. Solid and open symbols represent the products from round and dressed fish, respectively. As the concentration of $\kappa$-carrageenan increased, jelly strength of products from round and dressed sardine was increased markedly. As the concentration of $t$-carrageenan was increased, jelly strength of products from round and dressed sardine was increased moderately. As the concentration of $\lambda$-carrageenan was increased, jelly strength of products from round and dressed sardine was increased very gently. Generally, jelly strength of product from dressed sardine was larger than that of product from round sardine when the same carrageenan concentration was used. Breaking deformation of sardine terrine is shown in Fig. 3. When the concentration of $\kappa$-carrageenan was increased to 8 and $10 \%$, breaking deformation of product from round fish was slightly increased but breaking deformation of product from dressed fish was clearly decreased. When the concentration of t-carrageenan was increased up to $10 \%$, breaking deformation of products from round and dressed fish remained almost unchanged. As the concentration of $\lambda$ carrageenan was increased, breaking deformation of products from round and dressed fish was decreased.

The addition of $k$-carrageenan to mince from round sardine brought about the formation of hard texture in terrine from round sardine. The addition of $k$-carrageenan to mince from dressed sardine up to $6 \%$ brought about the formation of hard texture in terrine from dressed sardine, but the addition of 8 and $10 \% \kappa$-carrageenan brought about the formation of brittle texture in terrine from dressed sardine. The addition of tcarrageenan to minced sardine from round and dressed fish brought about the texture of moderate hardness in sardine terrine. The addition of $\lambda$-carrageenan to minced sardine from round and dressed fish brought about the formation of soft and brittle texture in sardine terrine.

\section{Force vs. Deformation Curve of Plunger Penetra-} tion Test and Result of Sensory Evaluation

The photographs of sardine terrine were shown in Fig. 4. The colour of terrine from round sardine was darker than that of terrine from dressed sardine, irrespective of type of carrageenan added. This is due to the fact that round sardine contains the viscera and dressed sardine does not. The moisture contents of sardine terrine were also shown in Fig. 4. They were about $65 \%$ in both products from round and dressed fish, irrespective of type of carrageenan added. The moisture contents of sardine terrine were not increased by the addition of $6 \%$ carrageenan.

Force vs. deformation curves (Fig. 5) were obtained on sardine terrine shown in Fig. 4. The patterns of force vs. deformation curve on sardine terrine with $6 \% \kappa$-carrageenan (both products from round and dressed fish) were similar to the patterns on Vienna sausage and fish sausage. Namely, the breaking deformation was small, and the drop of force after first peak was not sharp. Refer to our previous report. ${ }^{5)}$ In the patterns of force vs. deformation curve on sardine terrine with $6 \%$ c-carrageenan (both products from round and dressed fish), the breaking deformation was small, and the drop of force after first peak was very small. In the patterns of force vs. deformation curve on sardine terrine with $6 \% \lambda$ - 
Products from round fish
Products from dressed fish
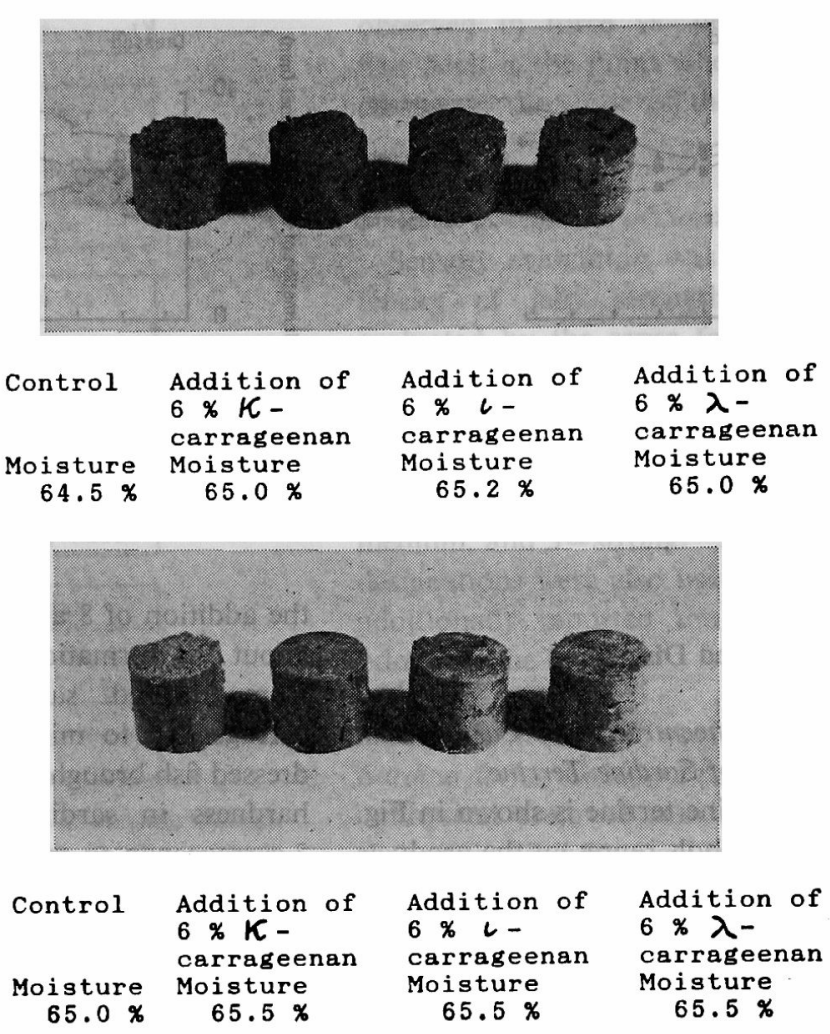

Fig. 4. Sardine terrines produced from round and dressed sardine.
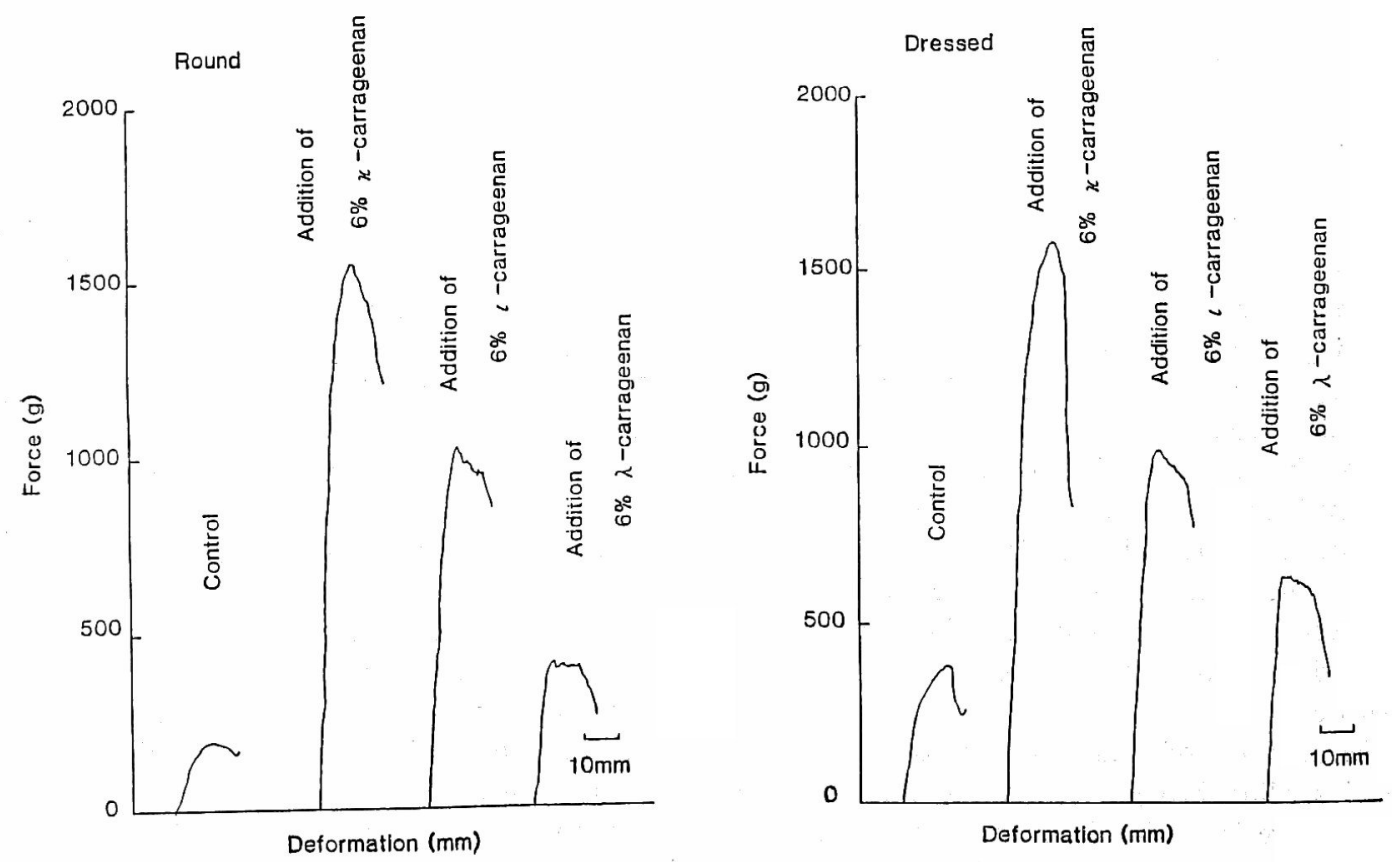

Fig. 5. Force vs. deformation curve of a ball-point plunger penetrating into sardine terrine.

The diameter of a ball-point plunger was $7 \mathrm{~mm}$. Force vs. deformation curves were obtained from sardine terrines shown in Fig. 4. 

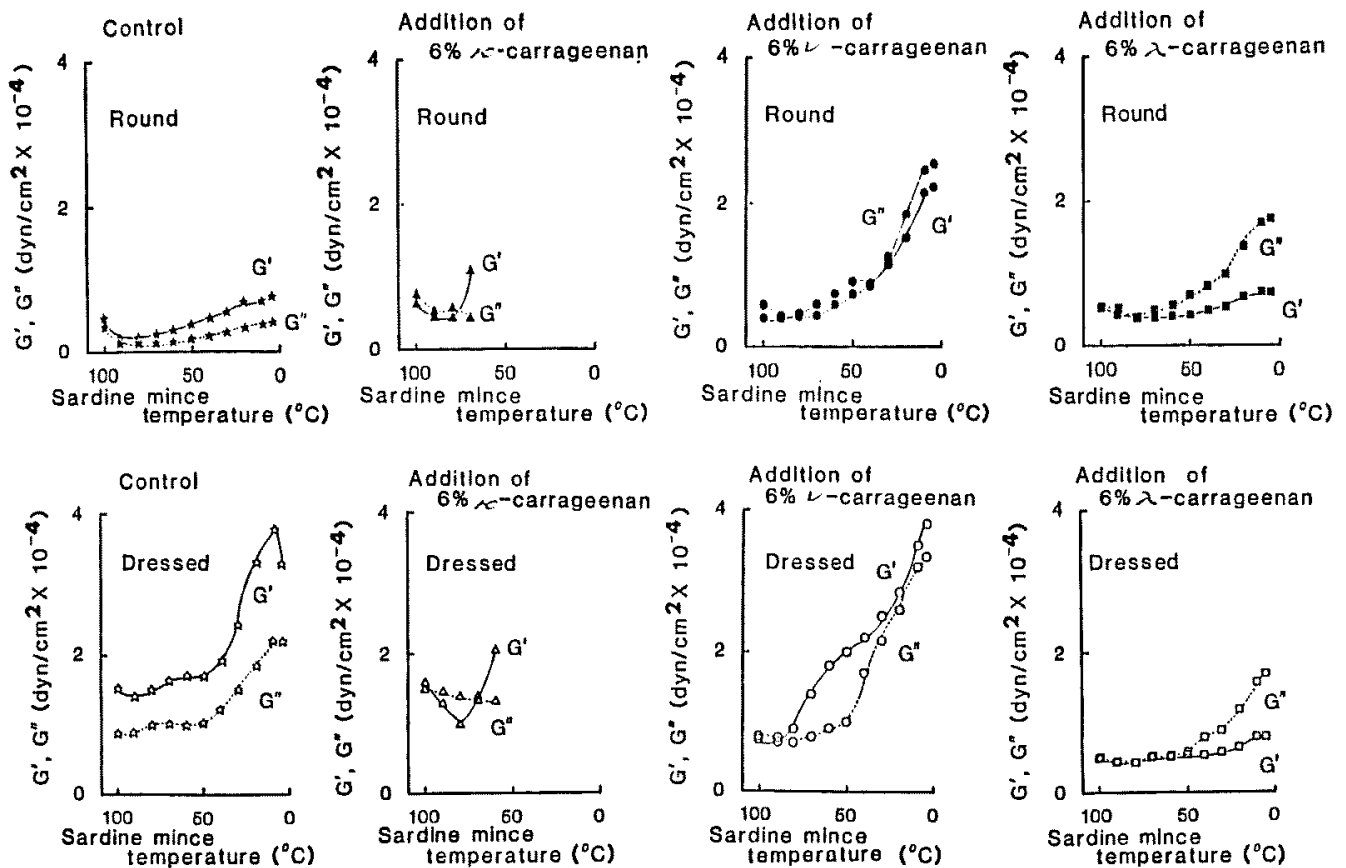

Fig. 6. Effect of carrageenan added on viscoelastic properties of minced sardine during cooling process.

Strain amplitude 0.10 . Frequency 3 cycle/s.

carrageenan (both products from round and dressed fish), the breaking deformation was small, and the obvious drop of force after first peak was not observed.

The sensory evaluation was also carried out on sardine products to which $6 \%$ carrageenan was added. As for the sensory evaluation, the sense intensity of jelly strength of products decreased in the order $\kappa^{-}, t^{-}$, and $\lambda$-carrageenan in the case of both products from round and dressed fish. Control was still weaker in the sense intensity than product with $\lambda$-carrageenan, in the case of both products from round and dressed fish. These results of the sensory evaluation corresponded to the instrumental values of jelly strength (Fig. 5). Panelists favored the products with $\kappa$-carrageenan and t-carrageenan in the case of both products from round and dressed fish. Among them, the product with $6 \%$-carrageenan from dressed fish was most favorable. This result would be due to the dropping of larger force after first peak. All sardine terrines were juicy. Fishy odour of products from dressed fish was weaker than that of products from round fish because the texture of the former was firmer and prevented the occurrence of fishy odour.
Viscoelastic Properties of Minced Sardine during Cooling Process

In the manufacturing process of sardine terrine, the temperature of minced sardine was once raised to $100^{\circ} \mathrm{C}$, then lowered to $5^{\circ} \mathrm{C}$. In this process, the addition of carrageenan was effective to jellify the minced sardine. This process was monitored with a model to investigate the relationship between the temperature and the viscoelastic properties. The result was shown in Fig. 6. In the case of mince without carrageenan from round sardine (round control), both $\mathrm{G}^{\prime}$ and $G^{\prime \prime}$ remained almost unchanged during the lowering of temperature to $5^{\circ} \mathrm{C}$. In the case of mince without carrageenan from dressed sardine (dressed control), both $\mathrm{G}^{\prime}$ and $\mathrm{G}^{\prime \prime}$ increased during the lowering of temperature below $50^{\circ} \mathrm{C}$. The values of $G^{\prime}$ and $G^{\prime \prime}$ were much larger in dressed control than in round control, when compared at any range of temperature.

In the case of mince from round or dressed sardine with $6 \% \kappa$-carrageenan, the lowering of temperature from $100^{\circ} \mathrm{C}$ to $80^{\circ} \mathrm{C}$ brought about the decrease of both $G^{\prime}$ and $G^{\prime \prime}$, probably due to the structural breakdown during the measurement of dynamic viscoelasticity (mechanical vibration). 
But the lowering of temperature from $80^{\circ} \mathrm{C}$ to $60^{\circ} \mathrm{C}$, increased $\mathrm{G}^{\prime}$ steeply because the jellification overcame the structure breakdown. The further lowering of temperature below $60^{\circ} \mathrm{C}$ brought about instantaneous large decrease of $\mathrm{G}^{\prime}$ due to the slip between the blade and the minced sardine jelly in the measuring apparatus. Therefore the measurement was discontinued.

In the case of mince from dressed sardine with $6 \%$ t-carrageenan, $\mathrm{G}^{\prime}$ increased steeply during the lowering of temperature below $80^{\circ} \mathrm{C}$, and $\mathrm{G}^{\prime \prime}$ did so during the lowering of temperature below $50^{\circ} \mathrm{C}$. In the case of mince from round sardine with $6 \%$ t-carrageenan, the changes of both $G^{\prime}$ and $\mathrm{G}^{\prime \prime}$ during the lowering of temperature were similar to those of mince from dressed sardine with $6 \%$ t-carrageenan. But the changes were clearer in mince from dressed sardine than in mince from round sardine.

In the case of mince from round or dressed sardine with $6 \% \lambda$-carrageenan, $\mathrm{G}^{\prime}$ did not change while $\mathrm{G}^{\prime \prime}$ increased gently in proportion to lowering of temperature.

From the above-mentioned result, we conclude that the gelation of minced sardine with $\kappa$ - or 6 carrageenan proceeded at the temperature below about $80^{\circ} \mathrm{C}$. The addition of $\lambda$-carrageenan to minced sardine did not induce the gelation caused by lowering of temperature. Therkelsen ${ }^{6)}$ elucidated the properties of $\kappa^{-,}, \mathrm{c}$, , and $\lambda$-carrageenans that the former two have the ability to enter into a helical structure to form a gel, but $\lambda$-carrageenan has not such ability. Therefore $\lambda$-carrageenan is used only as a food thickener. These respective properties of carrageenans were revealed further in the experiments using minced sardine as material. And the effects were more vivid when minces from dressed sardine were used.

\section{Acknowledgement}

We wish to thank to Mr. S. Hirata of Taiyo Kagaku Co., Ltd. for his valuable discussion and encouragement.

\section{References}

1) S. Ohta: Science of Cookery, 15, 171-178 (1982).

2) K. Hata, A. Sangajiri, and T. Fujita: Science of Cookery, 16, 155-160 (1983).

3) T. Nakayama, O. Mizuno, and E. Niwa: Nippon Suisan Gakkaishi, 51, 1675-1679 (1985).

4) T. Nakayama, K. Mitoh, S. Kanoh, and E. Niwa: Nippon Suisan Gakkaishi, 52, 1799-1805 (1986).

5) T. Nakayama, T. Ichikawa, S. Kanoh, and E. Niwa: Nippon Suisan Gakkaishi, 53, 2021-2030 (1987).

6) G. H. Therkelsen: in "Industrial Gums", 3rd ed., Academic Press, New York, 1985, pp. 103162.

7) D. A. Rees: Pure Appl. Chem., 53, 1-14 (1981),

8) E. R. Morris, D. A. Rees, and G. Robinson: $J$. Mol. Biol., 138, 349-362 (1980). 